\title{
The magnetic field of the pre-main sequence Herbig Ae star HD 190073^
}

\author{
C. Catala ${ }^{1}$, E. Alecian ${ }^{1}$, J.-F. Donati ${ }^{2}$, G. A. Wade ${ }^{3}$, J. D. Landstreet ${ }^{4}$, T. Böhm ${ }^{2}$, J.-C. Bouret ${ }^{5}$, S. Bagnulo ${ }^{6}$, \\ C. Folsom ${ }^{3,7}$, and J. Silvester ${ }^{3,7}$
}

1 Observatoire de Paris, LESIA, CNRS UMR 8109, 5 place Jules Janssen, 92195 Meudon Principal Cedex, France e-mail: claude.catala@obspm.fr

2 Laboratoire d'Astrophysique, Observatoire Midi-Pyrénées, 14 avenue Edouard Belin, 31400 Toulouse, France

Dept. of Physics, Royal Military College of Canada, PO Box 17000, Stn Forces, K7K 4B4, Kingston, Canada

4 Dept. of Physics \& Astronomy, University of Western Ontario, London, Canada N6A 3K7

5 Laboratoire d'Astrophysique de Marseille, Traverse du Siphon, BP 8, 13376 Marseille Cedex 12, France

${ }^{6}$ European Southern Observatory, Casilla 19001, Santiago 19, Chile

7 Department of Physics, Engineering Physics, and Astronomy, Queen's University, Kingston, ON K7L 3N6, Canada

Received 18 August 2006 / Accepted 13 October 2006

ABSTRACT

\begin{abstract}
Context. The general context of this paper is the study of magnetic fields in the pre-main sequence intermediate mass Herbig Ae/Be stars. Magnetic fields are likely to play an important role in pre-main sequence evolution at these masses, in particular in controlling the gains and losses of stellar angular momentum.

Aims. The particular aim of this paper is to announce the detection of a structured magnetic field in the Herbig Ae star HD 190073, and to discuss various scenarii for the geometry of the star, its environment and its magnetic field.

Methods. We have used the ESPaDOnS spectropolarimeter at CFHT in 2005 and 2006 to obtain high-resolution, high signal-to-noise circular polarization spectra which demonstrate unambiguously the presence of a magnetic field in the photosphere of this star.

Results. Nine circular polarization spectra were obtained, each one showing a clear Zeeman signature. This signature is suggestive of a magnetic field structured on large scales. The signature, which corresponds to a longitudinal magnetic field of $74 \pm 10 \mathrm{G}$, does not vary detectably on a one-year timeframe, indicating either an azimuthally symmetric field, a zero inclination angle between the rotation axis and the line of sight, or a very long rotation period. The optical spectrum of HD 190073 exhibits a large number of emission lines. We discuss the formation of these emission lines in the framework of a model involving a turbulent heated region at the base of the stellar wind, possibly powered by magnetic accretion.

Conclusions. This magnetic detection contributes an important new observational discovery which will aid our understanding of stellar magnetism at intermediate masses.
\end{abstract}

Key words. stars: activity - stars: magnetic fields - stars: rotation - techniques: spectroscopic -

stars: early-type - stars: pre-main sequence

\section{Introduction}

Magnetic fields can have a strong impact on pre-main sequence (PMS) evolution. In particular, the role of magnetic fields in the evolution of angular momentum during the PMS phase can be crucial, both by amplifying the angular momentum losses through stellar winds, and by coupling the stars with the massive accretion disks present around a large fraction of them. Magnetic fields are probably also important for evacuating angular momentum from the disks themselves in very early phases of star formation, impacting the initial conditions for pre-main sequence evolution.

In the case of the low mass PMS stars, the T Tauri stars, stellar magnetic fields are believed to channel the accretion flow toward the star's surface along magnetic lines, and to control the accretion disk structure in this magnetospheric accretion

* Based on observations obtained at the Canada-France-Hawaii Telescope (CFHT) which is operated by the National Research Council of Canada, the Institut National des Sciences de l'Univers of the Centre National de la Recherche Scientifique of France, and the University of Hawaii. region (Königl 1991; Paatz \& Camenzind 1996). The topology of this channelled accretion depends strongly on the magnetic field structure and the tilt angle between rotation and magnetic axis (Romanova et al. 2003). While evidence has been accumulated recently that a large fraction of the intermediate mass PMS stars, the Herbig Ae/Be stars, also host massive accretion disks (Grady 2005), the magnetospheric accretion scenario has not been investigated in detail in their case.

The search for magnetic fields in the Herbig Ae/Be stars and, if they exist, the detailed analysis of their strength and topology, are necessary steps in the study of PMS evolution and the interplay between the stars, their accretion phenomena and their winds.

Another fundamental reason for investigating magnetic fields in Herbig stars is related to our understanding of the strong globally-ordered magnetic fields observed in the chemically peculiar A and B stars (Ap/Bp stars). The most common interpretation, known as the primordial fossil field hypothesis, involves a magnification of interstellar magnetic field swept up during the process of star formation (Mestel 2001; Moss 2001). In this hypothesis, we expect to observe a fraction of the Herbig $\mathrm{Ae} / \mathrm{Be}$ 
stars which also host globally-ordered magnetic fields, representing the progenitors of the magnetic Ap/Bp stars.

Most Herbig Ae/Be stars show conspicuous signs of winds and activity. These active phenomena have often been assumed to be magnetic (Praderie et al. 1986; Catala et al. 1999), but no direct nor indisputable proof of this magnetic origin has been presented so far.

Spectropolarimetric observations have recently provided some new insight into the problem of magnetic fields of Herbig Ae/Be stars. After the pioneering measurement of an effective longitudinal field of about $50 \mathrm{G}$ in HD 104237 by Donati et al. (1997), using the UCLES spectrograph on the AAT, equipped with the visitor SemelPol polarimeter, Hubrig et al. (2004) announced the discovery of a magnetic field in the Herbig Ae star HD 139614, as well as 2 other marginal detections, using FORS1 in polarimetric mode on the VLT. This result was not confirmed by more recent, higher $S / N$ ratio observations with the ESPaDOnS spectropolarimeter at CFHT, which on the other hand provided clear magnetic detections for the Herbig Ae stars V380 Ori and HD 72106A (Wade et al. 2005). These authors also report the detection of a field in HD 101412, using FORS1 on the VLT.

Our knowledge of magnetic fields in Herbig stars is evolving rapidly. This paper constitutes another step in that direction, by presenting results of spectropolarimetric observations of one more Herbig star, HD 190073. This early-type star (A2IIIeB9IVep+sh, Pogodin et al. 2005) with many emission lines in its visible spectrum has been recently shown to be a young Herbig Ae/Be star (Cidale et al. 2000; de Winter et al. 2001). Its spectral energy distribution in the infrared, as well as its $9.7 \mu \mathrm{m}$ silicate feature, are very similar to those of well-known Herbig stars (Malfait et al. 1998; Chen et al. 2000). Its remarkably low projected rotational velocity $\left(v \sin i=9 \mathrm{~km} \mathrm{~s}^{-1}\right.$, Acke $\&$ Waelkens 2004) is rather unusual among Herbig Ae/Be stars, and can be indicative of either a very slow rotation, or a very small inclination of the rotation axis with respect to the line of sight. Recent interferometric observations in the infrared are best interpreted in terms of a circumstellar disk seen nearly face-on, although higher inclination angles cannot be ruled out by these observations, nor the interpretation of the interferometric visibilities as due to binarity (Eisner et al. 2004).

In Sect. 2, we present our spectropolarimetric observations and data reduction procedures. The results of our investigations are presented in Sect. 3. A conclusion is found in Sect. 4.

\section{Observations and data reduction}

We used the ESPaDOnS spectropolarimeter, recently installed on the $3.6 \mathrm{~m}$ Canada-France-Hawaii Telescope (Donati et al. 2006, in preparation), during 3 observing runs in 2005 partly devoted to Herbig Ae/Be stars. Table 1 presents the log of the observations.

The data were obtained in the polarimetric configuration of ESPaDOnS, yielding a spectral resolution of 65000 . All spectra were recorded as sequences of 4 individual subexposures taken in different configurations of the polarimeter, in order to yield a full circular polarization analysis, as described in Donati et al. (1997) and Donati et al. (2006, in preparation). No linear polarization analysis was performed. The data were reduced with the automatic reduction package "Libre-ESpRIT" installed at CFHT (Donati et al. 1997; Donati et al. 2006 in preparation). Stokes $I$ and Stokes $V$ spectra are obtained by proper combinations of the 4 subexposures, while check spectra, labelled $N$ spectra, are calculated by combining the subexposures in such a way to yield
Table 1. Journal of ESPaDOnS observations of HD 190073. The 5th column gives the $S / N$ ratio at $600 \mathrm{~nm}$ per spectral bin of $0.035 \mathrm{~nm}$, while the 6th column lists the $S / N$ ratio in the deconvolved LSD Stokes $V$ profile per velocity bin of $1.8 \mathrm{~km} \mathrm{~s}^{-1}$. The last column lists the resulting effective longitudinal magnetic field, calculated from the LSD profile using the complete line mask, as discussed in Sects. 2 and 3.3.

\begin{tabular}{lllllll}
\hline \hline $\begin{array}{l}\text { Date } \\
\text { dd/mm/yy }\end{array}$ & $\begin{array}{l}\text { JD } \\
(2453000+)\end{array}$ & UT & $\begin{array}{l}t_{\exp } \\
(\mathrm{s})\end{array}$ & $S / N$ & $\begin{array}{l}S / N \\
(\mathrm{LSD})\end{array}$ & $\begin{array}{l}B_{\text {eff }} \\
(\mathrm{G})\end{array}$ \\
\hline $22 / 05 / 05$ & 512.989 & $11: 40$ & 3290 & 370 & 3600 & $+69 \pm 15$ \\
$23 / 05 / 05$ & 513.969 & $11: 11$ & 3600 & 250 & 2500 & $+61 \pm 35$ \\
$24 / 05 / 05$ & 514.960 & $10: 59$ & 3600 & 400 & 3950 & $+68 \pm 17$ \\
$24 / 05 / 05$ & 515.065 & $13: 59$ & 2400 & 300 & 3100 & $+66 \pm 22$ \\
$25 / 05 / 05$ & 515.962 & $11: 01$ & 3600 & 370 & 3700 & $+64 \pm 16$ \\
$25 / 05 / 05$ & 516.088 & $14: 02$ & 2400 & 290 & 2950 & $+98 \pm 30$ \\
\hline $19 / 07 / 05$ & 570.916 & $09: 51$ & 1800 & 470 & 5300 & $+70 \pm 11$ \\
\hline $25 / 08 / 05$ & 607.789 & $06: 49$ & 2000 & 500 & 5500 & $+74 \pm 10$ \\
\hline $08 / 06 / 06$ & 895.921 & $10: 01$ & 2400 & 560 & 5900 & $+73 \pm 10$ \\
\hline
\end{tabular}

a null signal, that can be used to verify the reality of the signal measured in Stokes $V$.

The data of May 2005 are affected by a 1.3 mag loss compared to the other data presented here. This problem, which was due to damage to the external jacket of optical fibres, was fixed prior to the July run.

We subsequently applied the Least-Square Deconvolution (LSD) method described in Donati et al. (1997) to construct average photospheric profiles of both the $I$ and $V$ Stokes parameters. The LSD technique builds the average photospheric line profile by deconvolving the observed spectrum (both in $I$ and $V$ Stokes parameters, as well as for the null $N$ spectrum) from a line mask including all lines present in a synthetic spectrum of the star. The line mask was computed using a Kurucz Atlas 9 model with effective temperature, surface gravity and metallicity appropriate for HD 190073, as described in Table 2. In the deconvolution procedure, each line is weighted by the product of its $S / N$ ratio in the observed spectrum, its depth in the unbroadened Kurucz model, and its magnetic Landé factor. Hydrogen Balmer lines, strong He I lines, strong resonance lines, the Ca II IR triplet lines, as well as lines for which the magnetic Landé factor cannot be computed, were excluded from the mask, which otherwise contains all other lines whose depth in the model relative to the continuum is larger than 0.1 . In addition to this main line mask, we also constructed a sub-mask containing only lines whose relative depth in the Kurucz model is comprised between 0.1 and 0.4. We shall see later (Sect. 3.2) that these shallow lines have no or only very weak emission components, and this mask will therefore be used to study the purely photospheric contribution to the spectrum. The complete mask contains 1400 lines in the ESPaDOnS spectral domain, while the shallow-line mask has 1000 lines.

The LSD average line profiles were computed on a velocity grid with a $1.8 \mathrm{~km} \mathrm{~s}^{-1}$ sampling. The resulting relative noise in the LSD $V$ Stokes profiles is given in the 6th column of Table 1.

Finally, both non-magnetic and magnetic standard stars were observed with ESPaDOnS, allowing us to verify the nominal behaviour of the instrument (see Donati et al. 2006, in preparation, for details).

\section{Results}

\subsection{Fundamental parameters of HD 190073}

The effective temperature and surface gravity of HD 190073 were taken from Acke \& Waelkens (2004), as $T_{\text {eff }}=9250 \mathrm{~K}$ and 
Table 2. Fundamental parameters of HD 190073. See text for the range of $v \sin i$ and $v_{\text {macro }}$ values.

\begin{tabular}{|c|c|c|c|c|c|c|c|c|}
\hline $\begin{array}{l}T_{\text {eff }} \\
(\mathrm{K})\end{array}$ & $\begin{array}{l}\log g \\
\left(\mathrm{~cm} \mathrm{~s}^{-2}\right)\end{array}$ & $\overline{\overline{\log }\left(L / L_{\odot}\right)}$ & 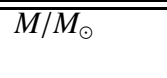 & 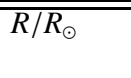 & $\begin{array}{l}\text { age } \\
\text { (Myr) }\end{array}$ & $\begin{array}{l}v \sin i \\
\left(\mathrm{~km} \mathrm{~s}^{-1}\right)\end{array}$ & $\begin{array}{l}v_{\text {macro }} \\
\left(\mathrm{km} \mathrm{s}^{-1}\right)\end{array}$ & $\begin{array}{l}v_{\text {rad }} \\
\left(\mathrm{km} \mathrm{s}^{-1}\right)\end{array}$ \\
\hline $9,250 \pm 250$ & $3.5 \pm 0.5$ & $1.92 \pm 0.12$ & $2.85 \pm 0.25$ & $3.6 \pm 0.5$ & $1.2 \pm 0.6$ & $0-8.6$ & $0-6.0$ & $0.21 \pm 0.1$ \\
\hline
\end{tabular}

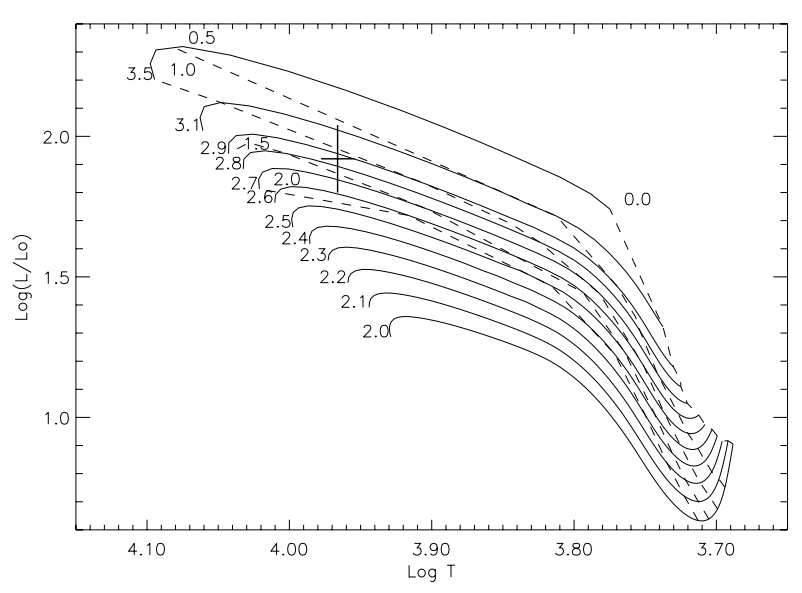

Fig. 1. The location of HD 190073 in the HR diagramme and the corresponding error bar are shown as a cross. Evolutionary tracks computed by CESAM are depicted in full lines and labelled by their masses. Isochrones are shown as dashed lines and labelled in Myr. Pre-main sequence evolutionary tracks start on the birthline and ages are counted using the birthline as time origin.

$\log g=3.5$ (cf. Table 2). The determination of its bolometric luminosity is made difficult by the fact that the Hipparcos parallax has a large error bar (Van den Ancker et al. 1998). Acke et al. (2005) adopt $\log \left(L / L_{\odot}\right)=1.92$, which is compatible with the lower limit of 1.80 determined by Van den Ancker et al. (1998). In this paper, we shall adopt the same value as Acke et al. (2005), and $\mathrm{a} \pm 0.12$ error bar to account for these difficulties.

We compared the location of HD 190073 in the HR diagramme to theoretical evolutionary tracks computed with the CESAM code (Morel 1997). This location is shown in Fig. 1, where evolutionary tracks for masses between 2 and $3.5 M_{\odot}$, and isochrones from 0 to $2 \mathrm{Myr}$ are shown. We assumed that PMS evolution started on the birthline in the HR diagramme, as defined by Palla \& Stahler (1990). Stellar ages are counted on each track using the birthline as time origin. Comparison of the location of HD 190073 in the HR diagramme with evolutionary tracks and isochrones yields estimates of its mass $\left(2.85 M_{\odot}\right)$ and age (1.2 Myr), and the corresponding CESAM models can be used to estimate its radius, $R=3.6 R_{\odot}$. The mass and radius of HD 190073 derived in this way yield a surface gravity $\log g=3.7$, to be compared to the value $\log g=3.5$ derived by Acke \& Waelkens (2004) from spectroscopy.

As described in Sect. 3.2, the spectrum of HD 190073 is characterized by the presence of numerous emission lines, on which photospheric absorption lines are superimposed. When performing the LSD deconvolution to determine the average line profile, in addition to the normal line mask, we also used the submask described in the previous section, containing only shallow lines with no or moderate emission, in order to study the photospheric line profiles without being perturbed by emission. Figure 2 shows the average line profiles computed with both masks, using the spectrum recorded in August 2005. We fitted this profile both with a simple Gaussian and with the convolution of a Gaussian and a rotation profile, calculated following

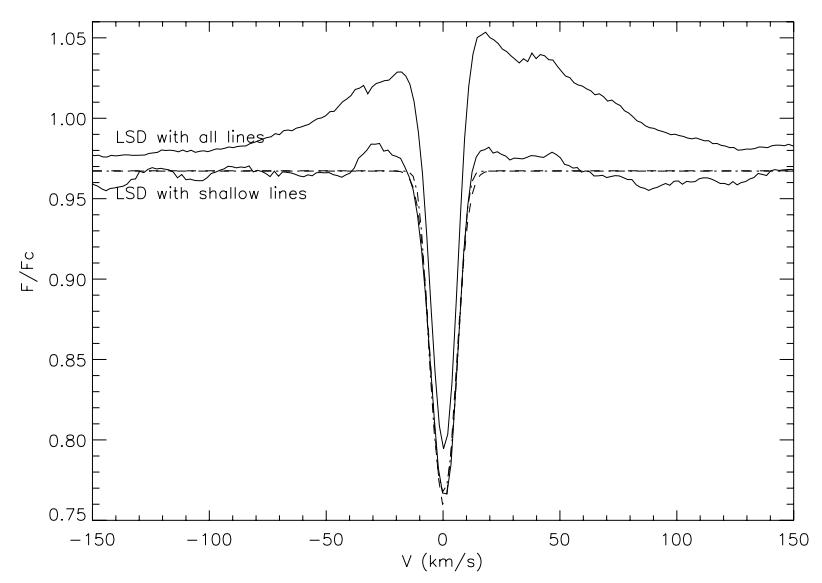

Fig. 2. The LSD average line profile of HD 190073 in August 2005. Average line profiles computed with both types of line masks described in the text are shown. The dashed line shows the Gaussian + rotation fit to the profile, while the dashed-dotted line corresponds to the purely Gaussian fit. Both models yield almost identical profiles.

Gray (1992). In the second case, the Gaussian involved in the convolution included contributions from instrument broadening, thermal broadening, and turbulence. We used an instrument broadening corresponding to a resolving power $R=65000$, adequate for ESPaDOnS, and a turbulent velocity of $2 \mathrm{~km} \mathrm{~s}^{-1}$.

Both types of fit are shown in Fig. 2. The Gaussian best fit yields a total line $F W H M$ of $12.4 \mathrm{~km} \mathrm{~s}^{-1}$, corresponding to a total turbulent velocity $v_{\text {turb }}$ of $6.8 \mathrm{~km} \mathrm{~s}^{-1}$ when instrumental broadening is taken into account ( $v_{\text {turb }}$ is defined throughout the paper as yielding a Gaussian broadening proportional to $\exp \left[-v^{2} / v_{\text {turb }}^{2}\right]$ ). The Gaussian+rotation fit implies a projected rotation velocity of $8.6 \mathrm{~km} \mathrm{~s}^{-1}$. We argue that both solutions are equally compatible with the data.

We note however that even the LSD profile constructed with the shallow-line mask includes some lines with a significant emission component, as well as lines where the photospheric absorption is filled-in by some emission, which can perturb the analysis of the line broadening. We therefore examined in detail a few lines which appear purely photospheric with no emission at all. The best examples of such lines are the O I lines near $616 \mathrm{~nm}$. In Fig. 3, we compare the profile of these lines with Kurucz Atlas 9 synthetic spectra, computed assuming the effective temperature and surface gravity listed in Table 2, and using detailed chemical abundances as determined by Acke \& Waelkens (2004). We computed two different series of Kurucz spectra: the first one includes a microturbulent velocity of $2 \mathrm{~km} \mathrm{~s}^{-1}$, an instrumental broadening corresponding to the spectral resolution of ESPaDOnS, and a rotational broadening left as a fitting variable; the second series of Kurucz spectra includes no rotation, a microturbulent velocity of $2 \mathrm{~km} \mathrm{~s}^{-1}$, and an isotropic macroturbulent velocity left as a free variable. We find that the $\mathrm{O} I$ lines can equally well be fitted either by a rotating model with $v \sin i=8.6 \mathrm{~km} \mathrm{~s}^{-1}$ and no macroturbulence, or by a model including a macroturbulent velocity of $6.0 \mathrm{~km} \mathrm{~s}^{-1}$ and no rotation. Appropriate combinations of rotation and 


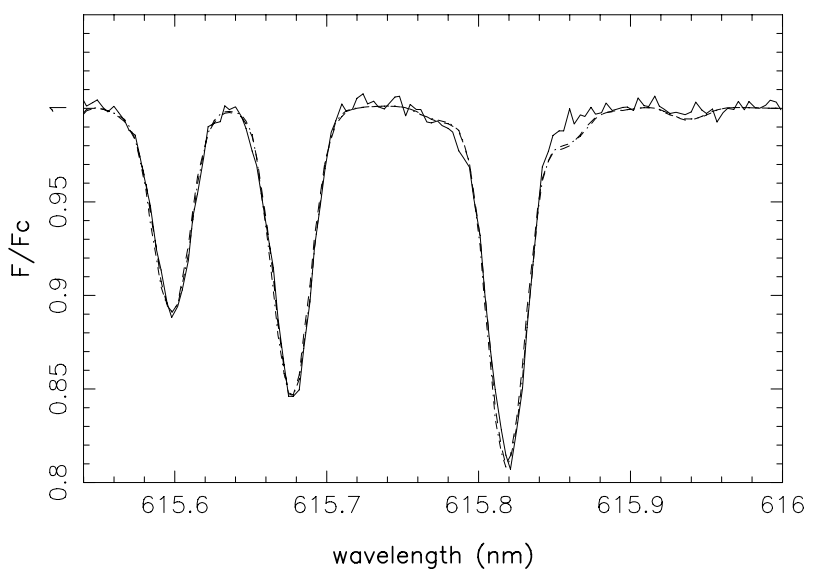

Fig. 3. The spectral region of the photospheric O I lines in August 2005. The solid line represents the observed profile, while the dashed line corresponds to the model with no rotation and a macroturbulent velocity of $6 \mathrm{~km} \mathrm{~s}^{-1}$, and the dashed-dotted line to a model with no macroturbulence and a projected rotation velocity of $8.6 \mathrm{~km} \mathrm{~s}^{-1}$. Both models yield almost identical profiles.

macroturbulence with intermediate values would of course fit the data as well.

We conclude from the above analysis that both rotating and macroturbulent models can equally reproduce the $\mathrm{O} I$ line profiles. This conclusion and the derived values of the projected rotation and macroturbulent velocities are in fair agreement with those derived from the analysis of the LSD profile. The examination of other lines in the spectrum led us to similar conclusions. Therefore, the projected rotation velocity can be anywhere between 0 and $8.6 \mathrm{~km} \mathrm{~s}^{-1}$, while the macroturbulent velocity can range from 0 to $6.0 \mathrm{~km} \mathrm{~s}^{-1}$. Although such high macroturbulent velocities may be surprising in an early A-type star like HD 190073, we note that significant photospheric turbulent velocities have been reported for main sequence A-type stars up to spectral type A0 (Landstreet 1998). LTE atmospheres are unstable to convection up to about $T_{\text {eff }}=10000 \mathrm{~K}$, due to the high opacity of excited and partly ionized hydrogen. Although this convection, which carries negligible heat, has no effect on the atmospheric structure, it still occurs and may result in photospheric turbulent velocity fields. In addition, accretion and mass loss, processes which are known to occur in very young stars like HD 190073, may also lead to additional photospheric turbulence. Hence the possibility of having photospheric turbulent velocities as high as $6 \mathrm{~km} \mathrm{~s}^{-1}$ in this star should be considered seriously.

We also attempted to use the Fourier transform of line profiles to disentangle turbulent velocity from rotation, following Reiners \& Schmitt (2003). We calculated the Fourier transform of the LSD average profile, as well as that of individual unblended photospheric lines, such as the O I lines discussed earlier. Unfortunately, we find that $v \sin i$ values lower than $9 \mathrm{~km} \mathrm{~s}^{-1}$ cannot be distinguished by this method, either with the LSD profile or with individual profiles, even with the high signal to noise ratio of our our data.

All derived fundamental parameters are summarized in Table 2.

\subsection{Unpolarized spectrum}

We repeatedly observed the Herbig Ae star HD 190073 with ESPaDOnS between May 2005 and June 2006. The star was observed up to twice a night for 4 consecutive nights in May, then additional isolated spectra were obtained in July and August 2005 and in June 2006 (see Table 1 for the log of observations). This observing strategy allows us to assess variability on a daily, monthly and yearly basis. The spectroscopic behaviour of HD 190073 was thoroughly studied by Pogodin et al. (2005), and we basically confirm their findings with our new observations.

The optical spectrum of HD 190073 is globally that of a star with $T_{\text {eff }}=9250 \mathrm{~K}$ and $\log g=3.5$, including a large number of emission lines. As a matter of fact, we find that many lines of ions such as Ca I, Ca II, Fe I, Fe II, Ti II, Si II, Sc II, Cr II, present in the Atlas 9 Kurucz synthetic spectrum adequate for HD 190073, having an intrinsic depth larger than 0.4 (i.e. the depth of the line in the synthetic spectrum in the absence of any broadening) exhibit emission in HD 190073.

All of these emission lines are superimposed on narrow absorption lines, with a width similar to that of the photospheric lines with no emission. The absorption lines and the absorption components overlapping the emission lines are in good agreement with the Kurucz synthetic spectrum. We therefore consider that these absorptions are of photospheric origin rather than due to a shell as discussed in Pogodin et al. (2005). The detailed analysis of Acke \& Waelkens (2004) indicates chemical abundances pretty close to solar, and our own synthesis using these abundances is in very good agreement with our observations, as shown for example by the fit of the O I lines in Fig. 3. This star therefore does not show the usual strong chemical peculiarities of Ap stars.

The emission components themselves have the same width all across the spectrum, equal to $65 \pm 7 \mathrm{~km} \mathrm{~s}^{-1}$ (FWHM). The intensity of these emission lines exhibits some moderate variability, of the order of a couple of percent in the one-year timeframe of our observations, while keeping the same shape and width. We also note that the emission lines have a centroid significantly redward of the absorption components that are superimposed on them, with a velocity variable between 4 and $19 \mathrm{~km} \mathrm{~s}^{-1}$, in an irregular manner from one epoch to another. Figure 4 shows an extract of the optical spectrum of HD 190073, compared to a Kurucz synthetic spectrum.

We also confirm the presence of strong P Cygni profiles at $\mathrm{H} \alpha$ and other hydrogen Balmer lines, with a strong monthly variability: between May and August 2005, although the red emission of these profiles remains moderately variable, we see a strong increase in the blueward absorption component. By June 2006, the absorption component of $\mathrm{H} \alpha$ has come back to its shape of May 2005. In contrast to this strong variability, the $\mathrm{H} \alpha$ emission component shows only moderate variations. This variability is shown in Fig. 5.

The most natural interpretation of the $\mathrm{H} \alpha$ P Cygni profile of HD 190073 involves a stellar wind. We have tentatively modelled this profile with a spherically symmetric wind model, similar to that of Bouret \& Catala (1998). This model includes a heated region at the base of the wind, surrounded by a cooler wind extending up to several tens of stellar radii. The coupled radiative transfer and statistical equilibrium equations for the hydrogen atom were solved in the comoving frame of the flow, using the Equivalent Two-Level Approach (ETLA) introduced by Mihalas \& Kunasz (1978) and used in Bouret \& Catala (1998). We find that the $\mathrm{H} \alpha$ P Cygni profile observed in August 2005 can be satisfactorily modelled with a wind having a mass loss rate $\dot{M}$ of $1.4 \pm 0.3 \times 10^{-8} M_{\odot} \mathrm{yr}^{-1}$ and a terminal velocity of $290 \pm 10 \mathrm{~km} \mathrm{~s}^{-1}$. The temperature of the model in the heated region at the base of the wind reaches $18000 \mathrm{~K}$, although we 

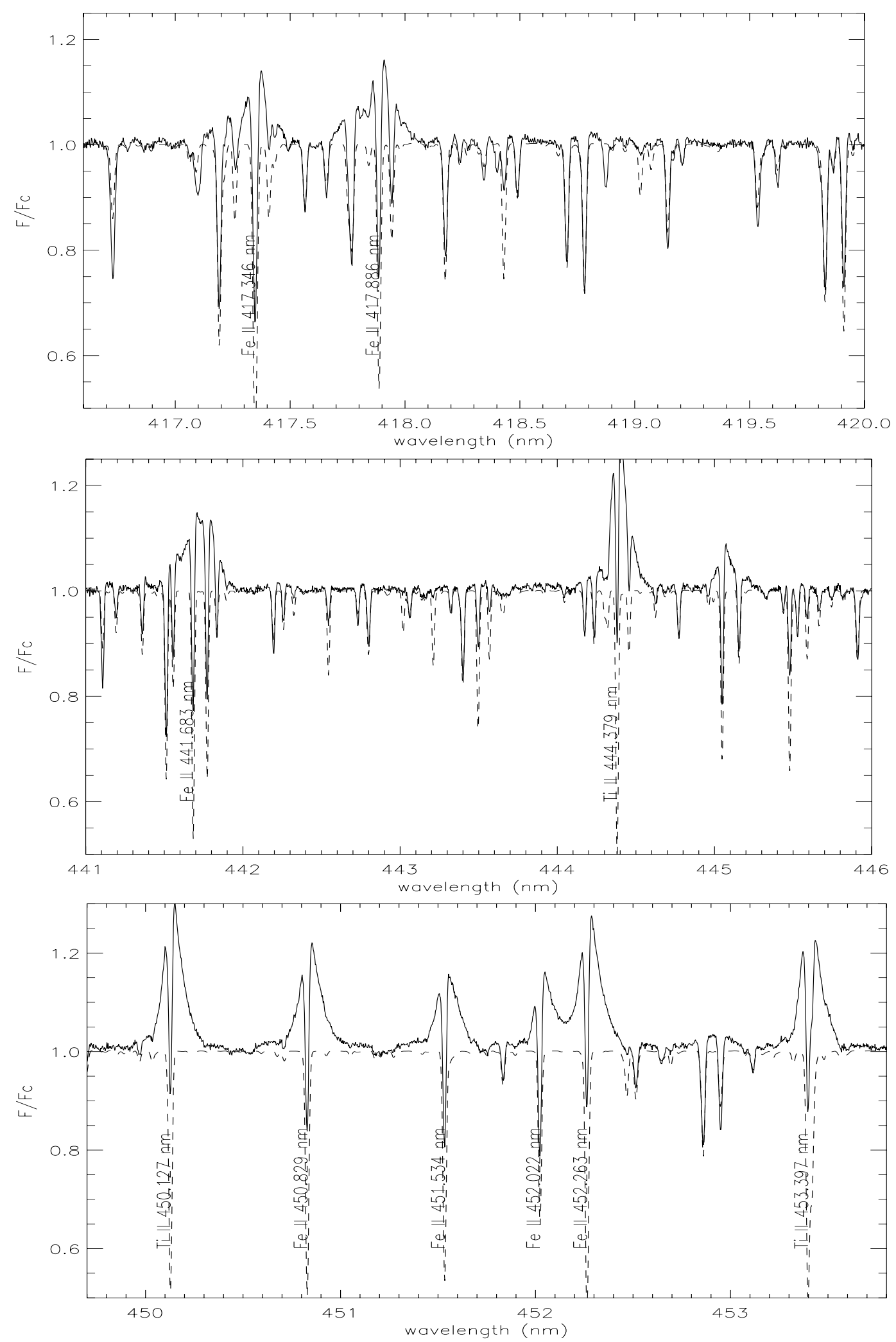

Fig. 4. Portions of the optical spectrum of HD 190073 in August 2005 (full line), compared to a synthetic spectrum computed from the Kurucz Atlas 9 model with $T_{\text {eff }}=9250 \mathrm{~K}, \log g=3.5,[\mathrm{Fe} / \mathrm{H}]=0, v \sin i=0 \mathrm{~km} \mathrm{~s}^{-1}, V_{\mathrm{r}}=0.21 \mathrm{~km} \mathrm{~s}^{-1}, V_{\text {micro }}=2.0 \mathrm{~km} \mathrm{~s}^{-1}$ and $V_{\text {macro }}=6.0 \mathrm{~km} \mathrm{~s}$ (dashed line). Note that faint lines are reasonably well reproduced by the synthetic model, while stronger lines show emission components.

cannot constrain it tightly with the $\mathrm{H} \alpha$ profile. The $\mathrm{H} \alpha$ line profile computed with this model in shown in Fig. 5. It fits the August 2005 observed profile reasonably well. We have not attempted to model the strong variability of the observed absorption component, which is presumably due to the presence of structures in the wind appearing on the line of sight. On the other hand, the global properties of the wind, e.g. the mass loss rate, remain constant, producing a more or less stable emission 


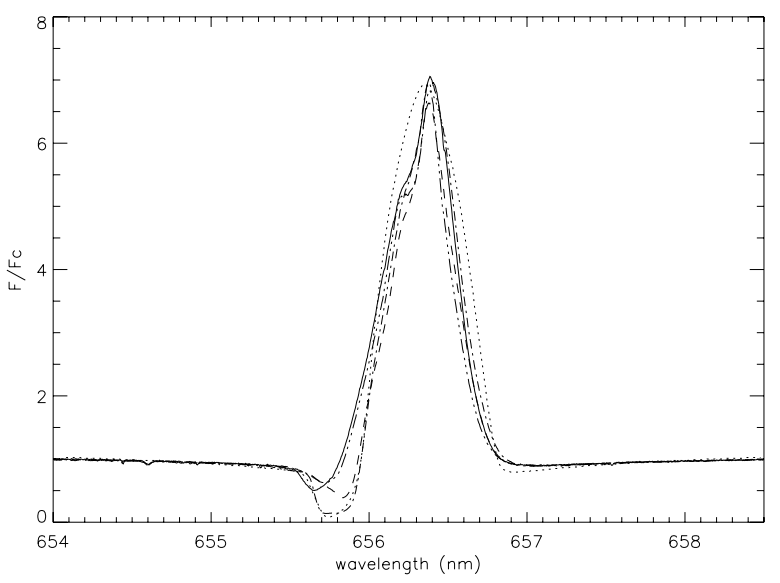

Fig. 5. The $\mathrm{H} \alpha$ P Cygni line profile of HD 190073 May 2005 (full line), July 2005 (dashed line), August 2005 (dashed-dotted line) and June 2006 (dashed-dot-dot-dotted line). Note the strong variation of the absorption component on a monthly basis, while the emission is only moderately variable. The computed profile fitted to the August 2005 data is shown as a dotted line.

component for this line (see Bouret \& Catala 1998, for a more detailed discussion). Note finally that the estimate of the mass loss rate given above assumes that all of the $\mathrm{H} \alpha$ emission originates from the wind. While the existence of a stellar wind is clearly demonstrated by the P Cygni profile at $\mathrm{H} \alpha$, other processes, such as emission from hot regions above the photosphere can contribute to this emission (see below), so that our estimate of $\dot{M}$ should be considered as an upper limit.

Quite in contrast with the $\mathrm{H} \alpha$ line, the Ca II $\mathrm{K} \& \mathrm{H}$ lines in our spectra do not show conspicuous variability in our one year timeframe, and are even very similar to the profiles presented by Pogodin et al. (2005), observed from 1994 to 2000.

\subsection{Magnetic field}

We detected a conspicuous Stokes $V$ signature in HD 190073, at all epochs in 2005 and 2006. This signature in $V / I$ has an amplitude of about $1 \times 10^{-3}$, and is presented in Fig. 6 . The shape of this Stokes $V$ signature is very simple, and indicates a globally structured magnetic field. The observed signature remains remarkably constant in all our observations from May 2005 to June 2006, and can be translated to an effective longitudinal magnetic field $B_{\text {eff }}$ of $+74 \pm 10 \mathrm{G}$, as measured on the August 2005 spectrum, using the approach described in Eq. (5) of Donati et al. (1997). The $\pm 10 \mathrm{G}$ error bar is calculated from the noise level of the August 2005 LSD Stokes $V$ profile, and the effective longitudinal magnetic fields measured from the other spectra of HD 190073 are in agreement with this value within the combined error bars. All $B_{\text {eff }}$ measurements are compiled in Table 1 .

We also calculated the standard deviation of the Stokes $V$ profile in time, for each velocity bin across the profile. We found it to be independent of velocity, with a value of the order of $2-3 \times 10^{-4}$, and with no noticeable feature at the location of the stellar line. This measured standard deviation in time is of the expected order of magnitude if there is no variation of the Stokes $V$ signature, considering the $S / N$ ratio in the LSD Stokes $V$ profiles listed in Table 1. We also noted that the standard deviation of the null $N$ profile has the same value as that of the Stokes $V$ profile. This stable Stokes $V$ signature implicitly requires a large scale magnetic field which is intrinsically stable on the timescale of our observations, i.e. over one year.

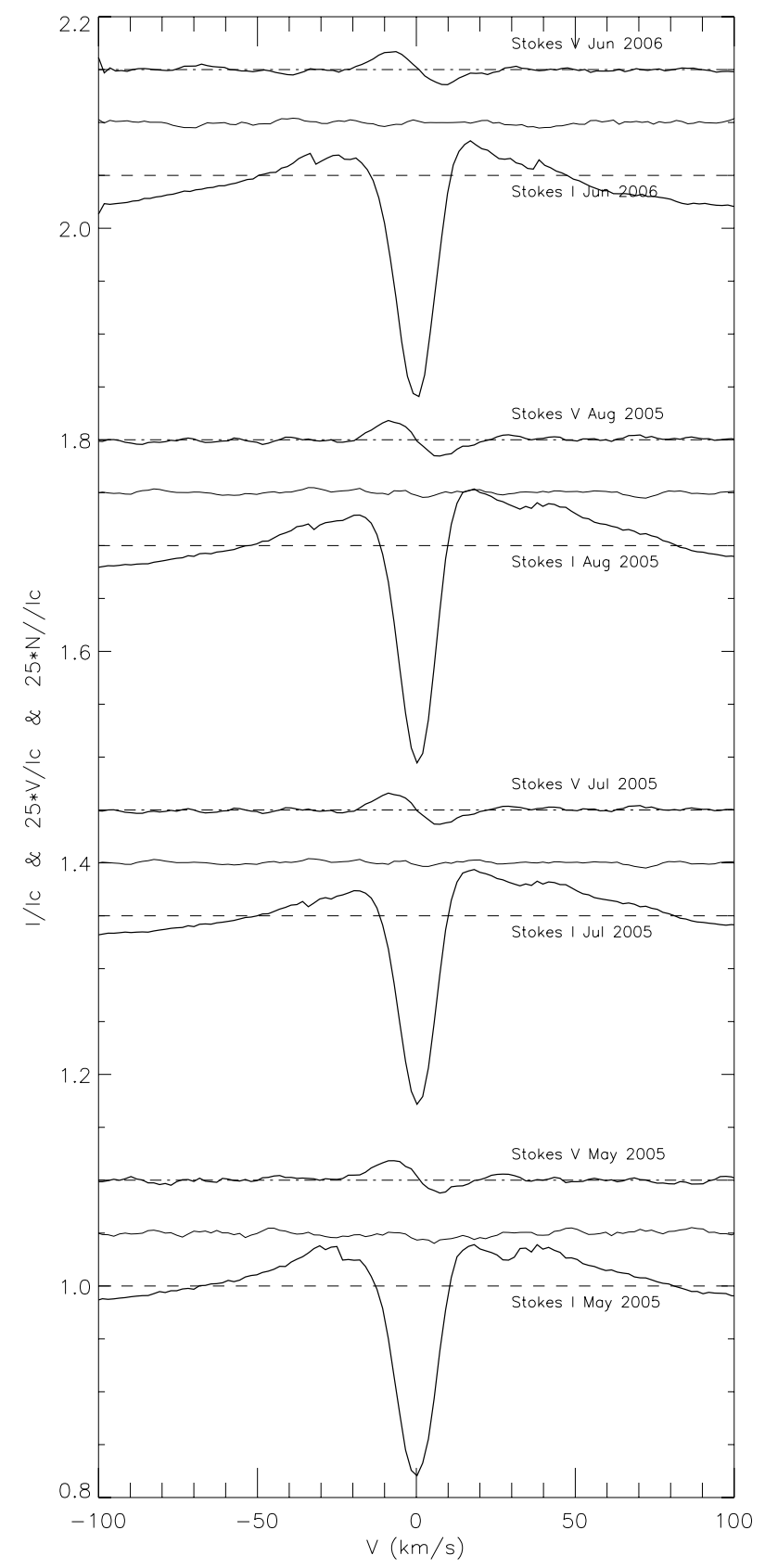

Fig. 6. LSD unpolarised and circularly polarised profiles of HD 190073 , at various epochs in 2005 and 2006. All LSD profiles are calculated with the mask including all photospheric lines. Spectra are shifted for display purposes. The thin curves appearing between the Stokes $I$ and $V$ profiles are the "null" spectra calculated as described in Sect. 2. The Stokes $V$ profiles and the null $N$ profiles are expanded by a factor 25 . The profile labelled "May 2005" is that of May 24, 10:59 UT. All spectra are in the heliocentric restframe.

Finally, the Stokes $V$ signature is detected on the LSD profiles calculated with both the full line mask and the shallow-line mask, with a very similar shape and intensity.

A first magnetic detection was reported long ago by Babcock (1958), which yielded an effective longitudinal magnetic field of $+270 \mathrm{G}$ for neutral species, while ionized species yielded a null magnetic field, and the Ca II H\&K lines indicated $-270 \mathrm{G}$. The large uncertainties in these earlier measurements, as reanalyzed by Preston (1969), imply that Babcock's results must 
be taken as simply hinting at the presence of a magnetic field rather than as a firm detection. This detection was not confirmed by Glagolevskij \& Chountonov (1998). Very recently, Hubrig et al. (2006), based on data obtained with the FORS1 instrument on the VLT, have reported a weak circular polarization signal in the $\mathrm{Ca}$ II H\&K lines, yielding an effective longitudinal magnetic field of the same order as the one we detect here on metallic lines.

We have tried to recover Babcock's result, by performing LSD analysis separately on lines of neutral and ionized species. We detect a clear Stokes $V$ signature in both cases, with no significant difference between them, nor compared to the Stokes $V$ signature calculated with all lines. We therefore do not confirm Babcock's results, either for the amplitude of the field, which is nearly 4 times weaker in our case, or for the difference between lines of neutral and ionized species. In addition, we see no Stokes $V$ signal in the Ca II H\&K lines, but the $S / N$ ratio of our spectra is low in that spectral region, resulting in a noise level of the order of $7 \times 10^{-3}$ in Stokes $V$ per spectral bin of $0.0025 \mathrm{~nm}$, which would not allow us to detect as weak a signal as seen in the LSD average, nor the level of signal reported by Hubrig et al. (2006).

The absence of variability of the magnetic signature in the course of one year can be interpreted in three different ways. Either the star is seen pole-on, and therefore whatever the magnetic configuration, the distribution of longitudinal field remains always the same; or the star is seen with an arbitrary inclination, but the magnetic field is symmetric about the rotation axis; or finally, the star and its magnetic field are in no particular configuration, but the rotation period is long enough compared to the one-year time span of our observations that no variation of the Stokes $V$ or $I$ profiles can be detected; a combination of the three interpretations is of course possible as well. Our estimate of $v \sin i$, which can be anything between 0 and $8.6 \mathrm{~km} \mathrm{~s}^{-1}$ is compatible with all three hypotheses.

A significant ambiguity about the rotation and its inclination angle has been discussed in the literature, in order to interpret the very low $v \sin i$ : the star was assumed to be either rotating very slowly intrinsically, or to be seen pole-on. We note that there is no consensus about this issue: while some authors prefer the intermediate inclination hypothesis on various and disputable theoretical grounds (Cuttela \& Ringuelet 1990; Pogodin et al. 2005), recent interferometric observations in the infrared may argue in favour of a face-on configuration for the extended circumstellar disk of HD 190073, implying a pole-on configuration for the star (Eisner et al. 2004).

In order to help resolve the ambiguity about the rotation of HD 190073, we searched for potential photometric variability of that star in the Hipparcos database. We find that the Hipparcos $H_{\mathrm{p}}$ magnitude of HD 190073 shows a scatter typically of the order of 0.02 mag on a daily time scale, with sporadic intervals where the daily scatter reaches 0.05 mag. This scatter is significantly larger than the usual error bar associated with Hipparcos $H_{\mathrm{p}}$ measurements, so we conclude that it must be due to intrinsic short term variability of the star. On the other hand, such a large short-term scatter does not allow us to detect longer term periodicity in the photometry that could be linked to rotation, although some hints exist for possible periodicities around 5.5 days and 22.3 days, but with amplitudes that are lower than the daily scatter of the data. These results are therefore ambiguous, and no conclusion can be drawn about the rotation period of HD 190073.

\subsection{Discussion}

We now discuss the nature of the wind, circumstellar environment and magnetic field of HD 190073 in the light of our observational results.

First, we have seen in Sect. 3.2 that the $\mathrm{H} \alpha$ line profile can be naturally explained by the presence of a stellar wind, with a mass loss rate of the order of $1.4 \times 10^{-8} M_{\odot} \mathrm{yr}^{-1}$, including a heated region at the base of the wind.

A basic question concerns the origin of the numerous emission lines. Because they all correspond to lines normally expected in the photosphere, they must be formed in a region where the density is not very different from that of the photosphere, otherwise the emission spectrum would show much more important differences with the photospheric absorption spectrum, in particular we would certainly see emission lines with no photospheric absorption counterpart, and conversely there would be many more strong photospheric absorption lines showing no emission.

The very rich emission spectrum of HD 190073, including all lines deeper than 0.4 in the photospheric line mask, indicates that the electron density of the heated region responsible for these emission lines is probably high, comparable to photospheric densities for this effective temperature and surface gravity, i.e. in the range $10^{13}-10^{14} \mathrm{~cm}^{-3}$. The temperature of this region must be significantly in excess of the effective temperature of the star, i.e. typically in the range $15000-20000 \mathrm{~K}$. These very rough estimates of the physical conditions in the heated region need to be verified and improved by a full non-LTE modelling of the formation of all observed spectral lines, which is outside of the scope of the present paper.

One possible assumption is that these emission lines are formed in a heated region located deep at the base of the wind, where such physical conditions could be met. We note that Cuttela \& Ringuelet (1990) suggest that a heated region is indeed present at the base of the wind, an assumption which is also supported by our tentative modelling of the $\mathrm{H} \alpha$ line. In this heated region at the base of the wind, which does not necessarily cover the whole stellar surface, the source function in spectral lines is increased compared to the rest of the atmosphere. Lines that are strong enough to be formed in this heated region at the base of the wind have a contribution in emission, as well as a contribution in absorption from the rest of the stellar surface. Weaker lines, which are formed deeper in the atmosphere, have no contribution from the heated region, presumably located too high above the star's surface. A heated region at the base of the wind has been frequently invoked to explain a few strong emission lines and lines of superionized species in the spectra of several Herbig Ae/Be stars (Catala \& Talavera 1984; Catala 1988; Bouret \& Catala 1998). In the case of HD 190073, where a much larger number of lines are seen in emission, the heated region just needs to be of higher density than in these other less extreme cases.

In order to produce symmetric emission lines as observed, the heated region must have a negligible velocity gradient. This is possible if the wind acceleration is gentle in this region, then becomes steeper higher up in the wind. The width of the symmetric, more or less Gaussian-shaped emission lines may be attributed to turbulent motions inside the heated region. A turbulent velocity of the order of $39 \mathrm{~km} \mathrm{~s}^{-1}$ would account for the observed $F W H M$ of $65 \mathrm{~km} \mathrm{~s}^{-1}$ of these lines. This turbulent velocity would be supersonic in the physical conditions of this heated region $(T \approx 15000-20000 \mathrm{~K})$. We note that supersonic turbulence has often been reported in the winds of hot stars, in 
particular in the case of PMS Herbig Ae/Be stars (see e.g. Bouret \& Catala 1998).

Although it is attractive, one problem with the interpretation of the emission lines in terms of a heated region at the base of the wind is that we would expect the emission lines to be centered in the star's rest frame, or even blueshifted if the outflow velocity in this region is not negligible, whereas we observe instead a variable but systematic redshift of the emission lines with respect to the photospheric absorption lines. Therefore, if this interpretation is correct, a more complex velocity pattern than a simple spherically symmetric wind must be assumed to account for this behaviour.

For instance, the heated region could be related to an accretion flow channelling matter from a circumstellar disk onto the star's surface in one or several magnetic funnels, in a similar way to what has been proposed for classical T Tauri stars (Paatz \& Camenzind 1996; Calvet \& Gullbring 1998; Romanova et al. 2003). The shocks produced by these accretion columns may be able to heat a sufficiently large region at the star's surface to produce the observed emission lines, which require temperatures and particle densities in the range 15000-20000 K and $10^{13}-10^{14} \mathrm{~cm}^{-3}$, respectively. Extrapolating the calculations of the accretion shock structure in classical T Tauri stars (Calvet \& Gullbring 1998) to the case of HD 190073, we suggest that these conditions can probably be met either in the preshock region, or in the photospheric region underlying the shocks. In the first hypothesis, a strong accretion rate is needed to provide the high particle densities required for the formation of the observed emission lines: still extrapolating the results of Calvet \& Gullbring (1998), and assuming a filling factor of the order of $1 \%$ for the shocked regions at the star's surface, we find that accretion rates of several $10^{-7} M_{\odot} \mathrm{yr}^{-1}$ are needed. In the second hypothesis, weaker accretion rates would probably be sufficient, although a full modelling of the photosphere perturbed by the shock, which is beyond the scope of this paper, would be necessary to study this issue.

Although the above interpretation of the line emission components is attractive, their width and their small variable redshift with respect to the absorption components remain to be explained. We may speculate that the kinetic energy of the accretion flow in the preshock region is partially transformed into turbulent energy, through flow instabilities, in order to explain the width of the emission components. On the other hand, the material being almost free falling in the preshock region, we would expect a much larger redshift than observed for the emission components, unless the angle between the accretion flow and the line of sight is systematically close to $90^{\circ}$. For instance, a simple configuration with an inclination angle $i \approx 0^{\circ}$ for the star's rotation axis (star seen pole-on) and an angle $\beta \approx 90^{\circ}$ between the rotation axis and the axis of a dipolar magnetic field, with matter being accreted onto the magnetic poles, would be compatible with the low $v \sin i$ and the width and redshift of the emission components if they are formed in the preshock region.

Detailed modelling of accretion flows in intermediate mass PMS stars, analogous to the work of Calvet \& Gullbring (1998) for classical T Tauri stars, is clearly needed in the future to study these issues in more details.

Alternative scenarios can be invoked for the formation of these emission lines. One possibility is that the emission lines are formed at the surface of a circumstellar disk, and are broadened by the disk's Keplerian rotation. In this case, we expect the emission components to exhibit a characteristic double-peak shape, which may resemble the observed profiles, for some particular choice of disk parameters. A Keplerian velocity at the surface of $V_{K}^{*}=390 \mathrm{~km} \mathrm{~s}^{-1}$ can be derived using the mass and radius of the star previously estimated, and the observed width of the emission lines $\left(65 \mathrm{~km} \mathrm{~s}^{-1} F W H M\right)$ implies that the inclination angle $i$ between the disk axis and the line of sight is such that $\sin i=0.083 \times\left(r_{\mathrm{em}} / R_{*}\right)^{1 / 2}$, where $R_{*}$ and $r_{\mathrm{em}}$ are respectively the stellar radius and the radius of the region of formation of the emission lines. This scenario would therefore imply that the system of HD 190073 and its disk is seen at small inclination, for reasonable values of $r_{\mathrm{em}}$ of a few stellar radii. The emission lines can be formed for instance by scattering of the stellar flux in an optically thin inner region of the disk, in a geometry similar to that suggested by Vink et al. (2005) for several other pre-main sequence stars. An important difficulty with this assumption, however, is that the emission lines all correspond to lines present in the photosphere, so that their region of formation in the disk must have density and temperature conditions which are not very different from those of the photosphere, as argued earlier. Such a coincidental similarity between photospheric and disk conditions is certainly unlikely. Also the systematic redshift between emission lines and photospheric absorptions remains unexplained in this scenario.

The ideas presented in this paper are clearly not sufficient to conclusively explain the formation of the observed emission components. More detailed studies would be needed to investigate the various hypothesis presented above. In particular further high quality spectropolarimetric data, and a full NLTE radiative transfer modelling of the observed lines should help us understand how these lines are formed, and constrain the configuration of the star, its magnetic field and circumstellar environment.

\section{Conclusion}

Our discovery of a globally structured magnetic field in HD 190073, a young $2.85 M_{\odot}$ Herbig Ae star, contributes an important piece to the puzzle of stellar magnetism for intermediate mass stars.

Our estimate of 1.2 Myr for its age indicates that stars less massive than $3 M_{\odot}$ can display significant surface magnetic fields at a very young age, in contradiction with Hubrig et al's (2000) conclusion that such stars become magnetic only after they have completed $30 \%$ of their main sequence life.

The star does not show the usual strong chemical peculiarities of Ap stars, possibly indicating that they develop on a time scale longer than $1 \mathrm{Myr}$, or that the accretion/mass loss processes that HD 190073 is currently experiencing are sufficient to disrupt their formation in the photosphere.

We were not able to constrain the geometry of HD 190073 and its magnetic field, due to the absence of variability of the polarimetric signature. Clearly a longer term investigation of this star should be undertaken.

Further spectropolarimetric monitoring of additional Herbig Ae and Be stars should also be performed, in order to detect other magnetic stars among them and to constrain their magnetic geometries and intensities. A statistical study of magnetism and rotation in pre-main sequence stars, in order to understand the evolution of magnetic fields and angular momentum during this early phase, would be of great value.

Acknowledgements. We warmly thank the CFHT staff for their efficient help during the observations. We are grateful to the referee, Dr. C. M. Johns-Krull, for his very useful comments which helped us improve this paper. G.A.W. acknowledges support from the Academic Research Programme of the Department of National Defence (Canada). G.A.W. and J.D.L. acknowledge support by the Natural Sciences and Engineering Council of Canada. 


\section{References}

Acke, B., \& Waelkens, C. 2004, A\&A, 427, 1009

Acke, B., van den Ancker, M. E., \& Dullemond, C. P. 2005, A\&A, 436, 209

Babcock, H. W. 1958, ApJS, 3, 141

Bouret, J.-C., \& Catala, C. 1998, A\&A, 340, 163

Calvet, N., \& Gullbring, E. 1998, ApJ, 509, 802

Catala, C. 1988, A\&A, 193, 222

Catala, C., \& Talavera, A. 1984, A\&A, 140,421

Catala, C., Donati, J. F., Böhm, T., et al. 1999, A\&A, 345, 884

Chen, P. S., Wang, X. H., \& He, J. H. 2000, Ap\&SS, 271, 259

Cidale, L., Zorec, J., \& Morrell, N. 2000, in IAU Colloq. 175, The Be Phenomenon in Early-Type Stars, ASP Conf. Ser., 214, 87

Cuttela, M., \& Ringuelet, A. E. 1990, MNRAS, 246, 20

de Winter, D., van den Ancker, M. E., Maira, A., et al. 2001, A\&A, 380, 609

Donati, J.-F., Semel, M., Carter, B. D., Rees, D. E., \& Cameron, A. C. 1997, MNRAS, 291, 658

Eisner, J. A., Lane, B. F., Hillenbrand, L. A., Akeson, R. L., \& Sargent, A. I. 2004, ApJ, 613, 1049

Glagolevskij, Y. V., \& Chountonov, G. A. 1998, Bull. Special Astrophys. Obs., 45,105

Grady, C. 2005, in The Nature and Evolution of Disks Around Hot Stars, ed. R. Ignace, \& K. G. Gayley, ASP Conf. Ser., 337, 155

Gray, D. F. 1992, The observation and analysis of stellar photospheres Cambridge Astrophysics Series (Cambridge: Cambridge University Press), 2nd edn., ISBN 0521403200
Hubrig, S., North, P., \& Mathys, G. 2000, ApJ, 539, 352

Hubrig, S., Schöller, M., \& Yudin, R. V. 2004, A\&A, 428, L1

Hubrig, S., Yudin, R. V., Schöller, M., \& Pogodin, M. A. 2006, A\&A, 446, 1089

Königl, A. 1991, ApJ, 370, L39

Landstreet, J. D. 1998, A\&A, 338, 1041

Malfait, K., Bogaert, E., \& Waelkens, C. 1998, A\&A, 331, 211

Mestel, L. 2001, ASP Conf. Ser., 205, 3

Mihalas, D., \& Kunasz, P. 1978, ApJ, 219, 635

Moss, D. 2001, ASP Conf. Ser., 248, 305

Morel, P. 1997, A\&AS, 124, 597

Paatz, G., \& Camenzind, M. 1996, A\&A, 308, 77

Palla, F., \& Stahler, S. 1990, ApJ, 360, L47

Pogodin, M., Miroshnichenko, A. S., Tarasov, A. E., et al. 2004, A\&A, 417, 715

Pogodin, M., Franco, G. A. P., \& Lopes, D. F. 2005, A\&A, 438, 239

Praderie, F., Simon, T., Catala, C., \& Boesgaard, A. M. 1986, ApJ, 303, 311

Reiners, A., \& Schmitt, J. H. M. M. 2003, A\&A, 398, 647

Romanova, M. M., Ustyugova, G. V., Koldoba, A. V., Wick, J. V., \& Lovelace, R. V. E. 2003, ApJ, 595, 1009

Van den Ancker, M. E., De Winter, D., \& Tjin A Djie, H. R. E. 1998, A\&A, 330, 145

Vink, J., Drew, J. E., Harries, T. J., Oudmaijer, R. D., \& Unruh, Y. 2005, MNRAS, 359, 1049

Wade, G. A., Drouin, D., Bagnulo, S., et al. 2005, A\&A, 442, L31 